\title{
Imaging flash lidar for safe landing on solar system bodies and spacecraft rendezvous and docking
}

\author{
Farzin Amzajerdian*a, Vincent E. Roback ${ }^{\mathrm{a}}$, Alexander E. Bulyshev ${ }^{\mathrm{b}}$, Paul F. Brewster ${ }^{\mathrm{a}}$, William \\ A. Carrion ${ }^{\mathrm{c}}$, Diego F. Pierrottet ${ }^{\mathrm{c}}$, Glenn D. Hines ${ }^{\mathrm{a}}$, Larry B. Petway ${ }^{\mathrm{a}}$, Bruce W. Barnes ${ }^{\mathrm{a}}$, Anna \\ M. Noe ${ }^{a}$ \\ aNASA Langley Research Center, Hampton, VA 23681; bAnalytical Mechanics Associates, \\ Hampton, VA 23666; 'Coherent Applications Inc., Hampton, VA 23666.
}

\begin{abstract}
NASA has been pursuing flash lidar technology for autonomous, safe landing on solar system bodies and for automated rendezvous and docking. During the final stages of the landing from about $1 \mathrm{~km}$ to $500 \mathrm{~m}$ above the ground, the flash lidar can generate 3-Dimensional images of the terrain to identify hazardous features such as craters, rocks, and steep slopes. The onboard flight computer can then use the 3-D map of terrain to guide the vehicle to a safe location. As an automated rendezvous and docking sensor, the flash lidar can provide relative range, velocity, and bearing from an approaching spacecraft to another spacecraft or a space station. NASA Langley Research Center has developed and demonstrated a flash lidar sensor system capable of generating 16k pixels range images with $7 \mathrm{~cm}$ precision, at $20 \mathrm{~Hz}$ frame rate, from a maximum slant range of $1800 \mathrm{~m}$ from the target area. This paper describes the lidar instrument and presents the results of recent flight tests onboard a rocket-propelled free-flyer vehicle (Morpheus) built by NASA Johnson Space Center. The flights were conducted at a simulated lunar terrain site, consisting of realistic hazard features and designated landing areas, built at NASA Kennedy Space Center specifically for this demonstration test. This paper also provides an overview of the plan for continued advancement of the flash lidar technology aimed at enhancing its performance to meet both landing and automated rendezvous and docking applications.
\end{abstract}

Keywords: Flash Lidar, Ladar, Laser Radar, ALHAT, Precision Navigation, Hazard Avoidance, Rendezvous and Docking

\section{INTRODUCTION}

Over the past decade, NASA has been actively advancing and testing 3-D flash lidar technology for two distinct applications: landing on solar system bodies1 and spacecraft rendezvous and docking with satellites or asteroids2. As part of a lander sensing system, a flash lidar generates 3-D terrain maps that are used to compare with onboard terrain maps to determine the lander's position relative to the intended landing site. Once the pre-designated landing site is found, the high resolution 3-D map is then used to identify hazardous terrain features and the safest landing location at the site. During final approach, the flash lidar can track terrain features and guide the vehicle to a safe landing location. This flash lidar sensor system is a solution for future robotic missions to the Moon and Mars that require landing at pre-designated sites of high scientific value, while avoiding hazardous terrain features, such as escarpments, craters, slopes, and rocks. Future missions planned to pave the path to colonization and mining of the Moon and human landing on Mars will need onboard hazard detection and precision navigation to ensure safe landing near previously deployed assets.

As an autonomous rendezvous and docking (AR\&D) sensor, our flash lidar technology and systems offer new capabilities for satellite servicing and space debris identification and removal. The system can be optimized to identify the rendezvous target and provide distance and bearing as the vehicle approaches the target satellite or station. Flash lidar is also viewed as a critical technology for asteroid mission concepts requiring precision rendezvous, identification of the landing or sampling site location, and navigation to the highly dynamic objects that may be tumbling in space.

NASA's interest in flash lidar technology stems from its ability to record full 3-D images with a single laser pulse, freezing the scene on every frame by removing all motion of the transmitter/receiver platform. Unlike earlier topographic imaging lidar systems that generated 3D images by scanning the laser beam across the scene and measuring the time of arrival for 
each returned laser pulse, the flash lidar records a full 3D image frame by illuminating the scene with a single laser pulse and imaging the scene onto one focal plane array (FPA). Each pixel in the FPA takes independent measurements of the lidar pulse time of flight to the target. Therefore, the flash lidar permits much higher frame rates without any blurring or inaccuracies due to the platform motion.

The effectiveness of flash lidar for AR\&D application was tested on three Space Shuttle flights before it retired. These demonstration flights to International Space Station were SpaceX DragonEye in 2009 and 2011 (STS-127 and 133), and Sensor Test for Orion Rel-Nav Risk Mitigation (STORRM) in 2011 (STS134). In these flights, the ability of the flash lidar to provide relative range and bearing information to the spacecraft was demonstrated. The flash lidar landing application was demonstrated through a closed-loop flight test onboard Morpheus free-flyer vehicle under the Autonomous Precision Landing and Hazard Avoidance Technology (ALHAT) project. After extensive AR\&D and landing demonstration tests at NASA, we continue to advance this promising technology in order to meet all the desired performance goals, to reduce its size, mass, and power, and to improve its robustness for operation in space environment.

\section{OPERATIONAL CONCEPT}

\subsection{Landing Operation}

As a landing sensor, flash lidar can perform four essential functions during descent and landing phases: Altimetry, Terrain Relative Navigation (TRN), Hazard Detection and Avoidance (HDA), and Hazard Relative Navigation (HRN) ${ }^{3-5}$. Fig. 1 illustrates flash lidar operation in the context of lunar landing. The lidar begins its operation at about $20 \mathrm{~km}$ above the ground after the thruster rocket firing initiated. At this stage, the lidar transmitter beam is focused to illuminate only a few pixels in the center of the detector array to measure range to the ground. Reducing the divergence of the lidar transmitter beam to a fraction of its receiver field of view increases its operational slant range to well over $20 \mathrm{~km}$ from a nominal 2 $\mathrm{km}$. The ground-relative altitude measurements provided by the flash lidar reduces the vehicle position error significantly since the Inertial Measurement Unit (IMU) suffers from drastic drift over the travel time from the Earth. The IMU drift error can be over $1 \mathrm{~km}$ for a Moon-bound vehicle and over $10 \mathrm{~km}$ for Mars. Accurate altitude data reduces position error to a few hundred meters. When the altitude drops to about $15 \mathrm{~km}$, the lidar beam is expanded to illuminate a larger number of detector pixels of the order a thousand pixels. In this phase of its operation, the flash lidar generates relatively lowresolution elevation data of the terrain below that will be compared with stored maps having known surface features such as large craters. This process, referred to as Terrain Relative Navigation, further reduces the vehicle's relative position error from hundreds of meters to tens of meters. From about $1 \mathrm{~km}$ to $0.5 \mathrm{~km}$ altitude, the flash lidar operates with its full field of view, generating a high resolution elevation map of the landing area while identifying hazardous features such as rocks, craters, and steep slopes. This elevation map is then processed to determine the most suitable landing location (HDA function). The flash lidar then continues to update the map in order to establish a trajectory toward the selected landing location. This phase of flash lidar operation is referred to as Hazard Relative Navigation. The flash lidar operation terminates at approximately $100 \mathrm{~m}$ above the ground before the vehicle thrusters create a dust plume.

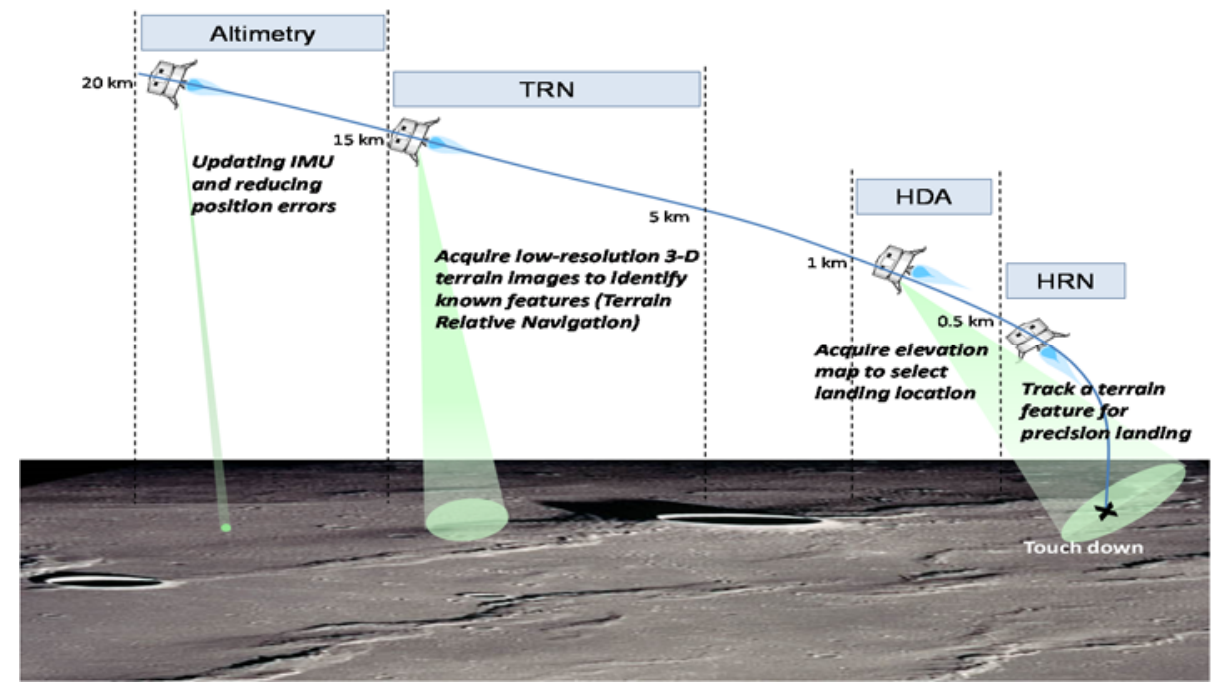

Figure 1. Lunar landing scenario. 
Mars operational scenario is very similar to lunar landing except that the lidar may not be able to begin its operation until after the vehicle velocity is reduced to well below hypersonic speeds by parachutes or deployable decelerators and the heat shield is released. In this case, the altimetry and TRN functions are initiated at the same time. Table 1 lists the nominal altitudes associated with each of flash lidar functions for lunar and Mars landing. It is noted that the lidar operational altitude may be significantly different from its operational slant range depending on the vehicle flight trajectory and the lidar pointing angle. During the HDA phase, the actual slant path to the ground can be more than 1.5km and the lidar incident beam angle relative to the surface can be less than 45 degrees depending on the flight trajectory. Therefore, the flash lidar shall have a maximum operational range of at least $2 \mathrm{~km}$ with fully illuminated focal plane array at normal incidence laser beam angle with respect to the ground.

Table 1. Flash lidar functions with corresponding nominal operational altitudes.

\begin{tabular}{|l|c|c|}
\hline \multirow{2}{*}{ Function } & \multicolumn{2}{|c|}{ Nominal Operational Altitude Range } \\
\cline { 2 - 3 } & Lunar Landing & Mars Landing \\
\hline HDA/HRN & $1000 \mathrm{~m}-100 \mathrm{~m}$ & $1000 \mathrm{~m}-100 \mathrm{~m}$ \\
\hline TRN & $15 \mathrm{~km}-5 \mathrm{~km}$ & $10 \mathrm{~km}-3 \mathrm{~km}$ \\
\hline Altimetry & $20 \mathrm{~km}-100 \mathrm{~m}$ & $10 \mathrm{~km}-100 \mathrm{~m}$ \\
\hline
\end{tabular}

\subsection{Autonomous Rendezvous and Docking Operation}

NASA is interested in developing a common capability for a wide range of missions requiring Rendezvous Proximity Operations and Docking (RPOD). These missions include human landing on the Moon and Mars, lunar mining, crew and supply transportation to and from the International Space Station, satellite servicing, space debris removal, and asteroid sample return and redirect (Fig. 2). The common capability implies that system must be: 1) fully autonomous, meaning RPOD can be executed onboard without ground support; and 2) fully operational with both cooperative and noncooperative targets. Non-cooperative targets means no RF transmitters, optical reflectors, or pre-installed distinguishing markings on the targeted body ${ }^{2,6}$. A flash lidar based solution will be a major component of the AR\&D system due to its ability to identify the docking location on the targeted body (,either man-made platforms or asteroids,) and to provide the necessary bearing, range, and relative attitude data for executing the rendezvous and docking maneuver ${ }^{7}$. Flash lidar operational scenario for AR\&D is similar to that of the landing application. The main difference is the size of the target body, which may be smaller than the lidar field of regard for the AR\&D application. The flash lidar starts its operation from tens of kilometers away using a few of its pixels to determine the distance to the target. The flash lidar will then operate in full field of view from $2 \mathrm{~km}-3 \mathrm{~km}$ distance to characterize the target surface and identify the docking location. Below $1 \mathrm{~km}$, the flash lidar provides direct range measurements to the target, along with 3D range images that are used to compute the vehicle relative pose for use by the navigation filter.
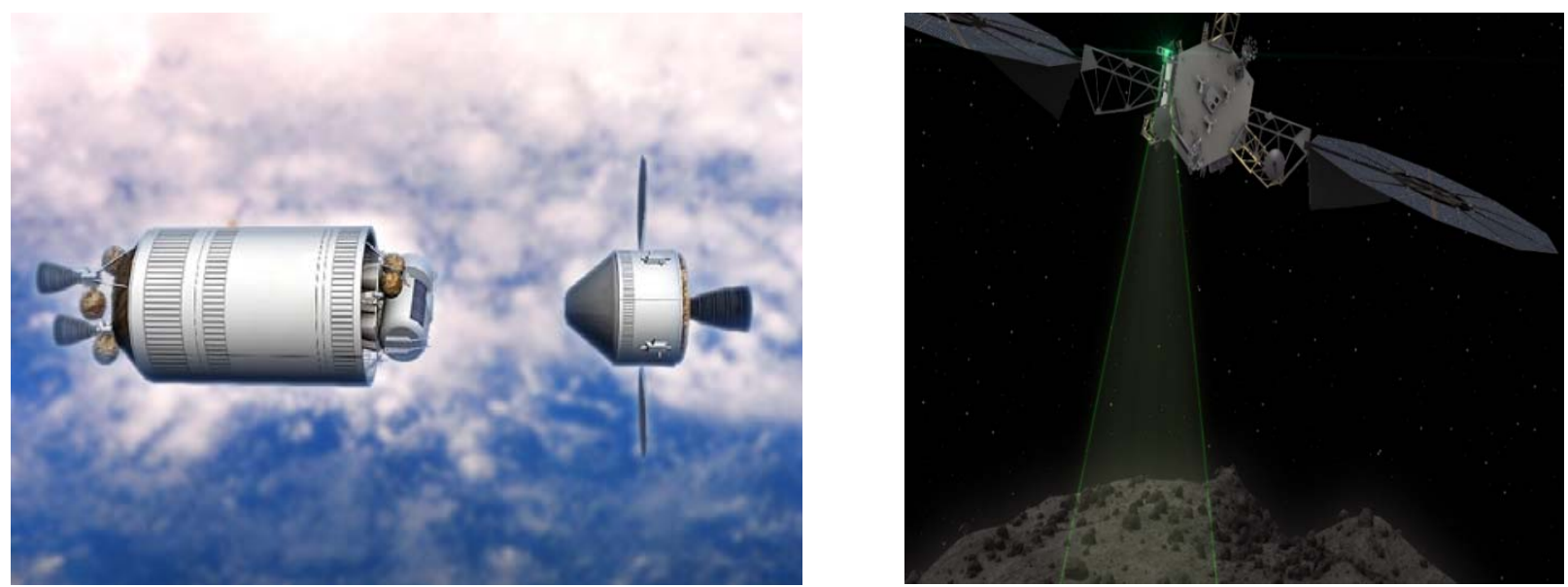

Figure 2. a) Orion vehicle docking with an Earth Departure Stage; b) Lidar sensor characterizing an asteroid surface before terminal approach for collecting samples or capturing a boulder. 


\section{ALHAT FLASH LIDAR SENSOR SYSTEM}

The flash lidar is one of three lidar sensors developed at NASA Langley Research Center (LaRC) for the ALHAT project. The other two sensors are a long range laser altimeter capable of high resolution range measurement $(5 \mathrm{~cm})$ from up to 50 $\mathrm{km}$ altitude and a Doppler lidar capable of providing precision vector velocity and altitude $(<1 \mathrm{~cm} / \mathrm{sec}$ and $30 \mathrm{~cm}$ respectively) from $3 \mathrm{~km}$ altitude. The flash lidar uses a 3-D imaging camera, also referred to as the sensor engine, developed by Advanced Scientific Concepts (ASC) ${ }^{8,9}$. The sensor engine consists of a detector array integrated with a matching ROIC, the associated detector/ROIC power supply, control electronics, and a real-time processor that calibrates the output of the ROIC and outputs both range and intensity image frames. The ASC sensor engine has a 128x128 pixel array that is capable of generating range images with $7 \mathrm{~cm}$ precision at up to 30 frames per second. Fig. 3 shows the prototype flash lidar consisting of a sensor head and an electronics chassis. The sensor head houses the sensor engine, the transmitter laser, and the transmit/receive optics, while the electronics box houses the sensor Controller and Data Handling (C\&DH) unit, the laser driver, the power conditioning/distribution unit, and temperature control boards. The transmitter laser, developed by Fibertek, generates $50 \mathrm{~mJ}$ pulses at 1.06 micron wavelength. The laser output beam has a uniform square shaped beam matching the detector array. The lidar C\&DH unit performs a number of functions including controlling and monitoring various lidar components, interfacing with avionics, and performing image processing and conditioning. The C\&DH executes a median filter algorithm for eliminating sporadic range noise, masks the known bad pixels, and applies an absolute range calibration routine. Flash lidar is able to detect hazardous terrain features as small as $30 \mathrm{~cm}$ from about 1.8 $\mathrm{km}$ of distance when its field of view is adjusted for $10 \mathrm{~cm}$ spatial resolution. The maximum operational distance of the flash lidar can be extended by reducing the divergence of its transmitter laser and illuminating a subset of its detector pixels. Assuming no atmospheric attenuation, the maximum operational range of the lidar is inversely proportional to the number of illuminated pixel squared. For example, the lidar's operational range increases from $1800 \mathrm{~m}$ to $20 \mathrm{~km}$ by reducing the number of illuminated pixels from $128 \times 128$ to 11 x 11 . Therefore, this flash lidar can provide useful low resolution 3-D images of the terrain and altitude data even during the initial descent phase.
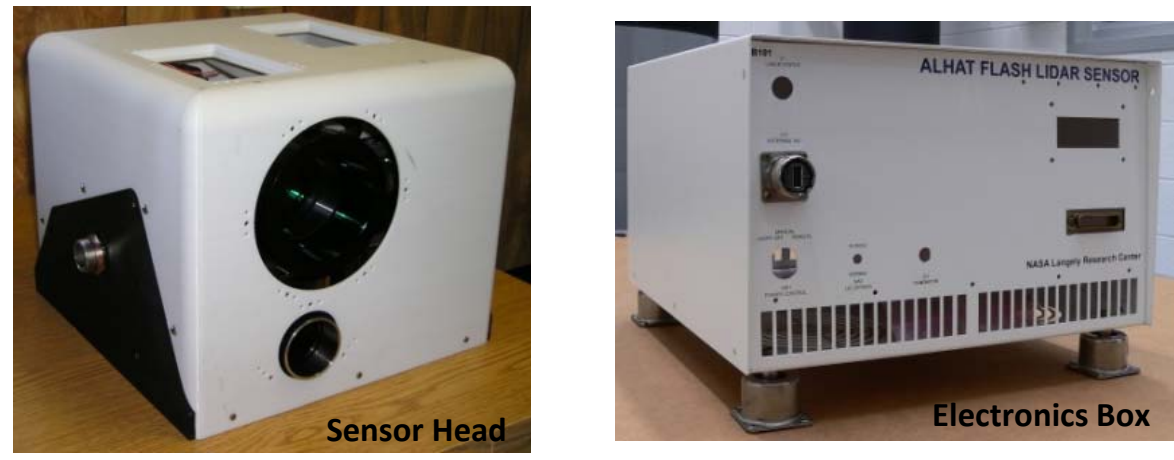

Figure 3. ALHAT Flash Lidar Sensor.

\section{ALHAT DEMONSTRATION FLIGHT TESTS}

The capabilities of the flash lidar along with the other two lidar sensors ${ }^{10}$ (Doppler Lidar and Laser Altimeter) mentioned earlier were assessed and their performance were characterized during different phases of their development through extensive testing at the NASA-LaRC Lidar Test Range facility and during five helicopter and fixed-wing aircraft flight test campaigns. The first four flight test campaigns provided invaluable data for the development of fully autonomous prototype systems. The prototype lidars were integrated with other ALHAT subsystems ${ }^{1,11}$ and tested in one last helicopter flight test before closed-loop demonstration using the rocket-powered Morpheus vehicle. The ALHAT subsystems included Hazard Detection System (HDS) ${ }^{12}$ and Autonomous Navigation System ${ }^{13}$. HDS uses the flash lidar images to generate a digital elevation map of the landing area and to subsequently select the best landing location given the vehicle's constraints and the mission objectives. The Navigation System uses the velocity and altitude data from the Doppler Lidar and Laser Altimeter to precisely determine the vehicle position and navigate the vehicle to the safe landing site provided by HDS. The last helicopter test campaign ${ }^{14,15}$ was aimed at testing the complete ALHAT landing system with the prototype sensors operating in concert with the HDS and the Navigation System in preparation for Morpheus closed-loop demonstration flights. Figure 4 shows the lidar sensors mounted beneath the helicopter. 


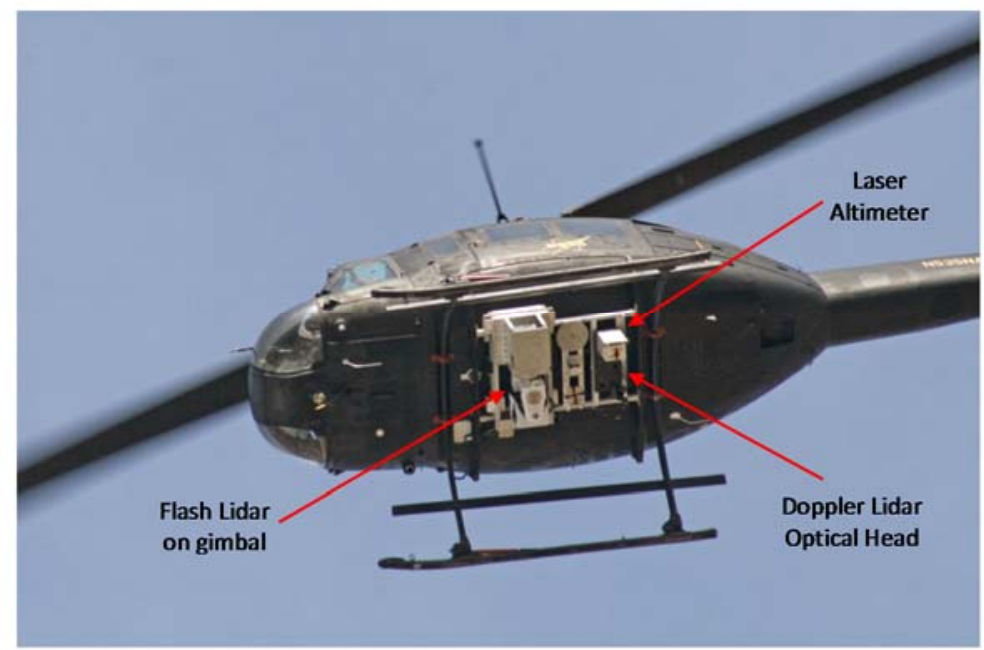

Figure 4. Prototype lidar sensors mounted under a Huey helicopter during Field Test 5. The flash lidar and Doppler Lidar electronic chassis were placed in a rack inside the cabin. The flight test was conducted at NASA Kennedy Space Center over a hazard field constructed near the Space Shuttle Landing Facility.

Fig. 5 shows the flash lidar along with the Doppler lidar and laser altimeter on the Morpheus vehicle. Morpheus ${ }^{16}$ is a rocket-powered, terrestrial flight-test vehicle built by NASA Johnson Space Center (JSC) to demonstrate advanced propulsion and GN\&C technologies for future landing missions. A series of integration tests were conducted to validate sensor interfaces and operational procedures ${ }^{17}$. These integration tests included three tethered tests during which the lidars were activated to communicate and provide data to various avionics systems while the Morpheus vehicle was suspended from a crane and executed a controlled flight procedure. The Morpheus flights were at a hazard field specifically constructed for this purpose near the north end of the Shuttle Landing Facility (SLF) runway at NASA Kennedy Space Center (Fig. 6). The hazard field is a $100 \mathrm{~m} \times 100 \mathrm{~m}$ area simulating a challenging lunar terrain and consists of realistic hazard features (rock piles and craters) and designated landing areas ${ }^{18}$.
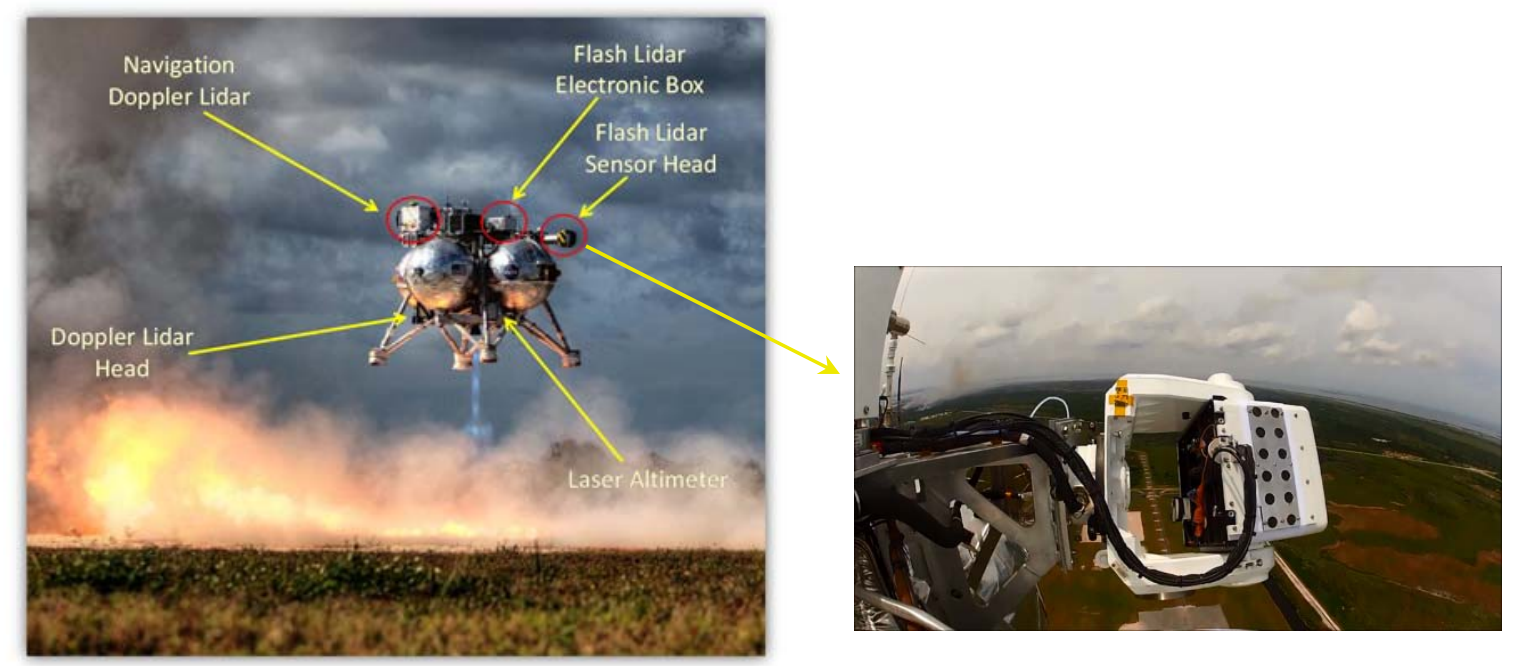

Figure 5. Lidar sensors integrated onto rocket-propelled Morpheus vehicle.

The flight profile, as shown in Fig. 6, is designed to demonstrate the autonomous safe landing system that controls the vehicle flight trajectory to the selected safe site and executes a landing maneuver using the lidar sensors' data. Morpheus launches from a pad next to the SLF runway and climbs to $250 \mathrm{~m}$ and then travels toward the hazard field about $500 \mathrm{~m}$ downrange. A few seconds after the vehicle begins its descend trajectory, the flash lidar maps the hazard field and provides it to HDS to identify the safe landing locations and selects the best one. Once the coordinates of the landing location is 
provided to the navigation system, the vehicle uses the Laser Altimeter and Doppler Lidar to precisely navigate to the selected location within the hazard field.

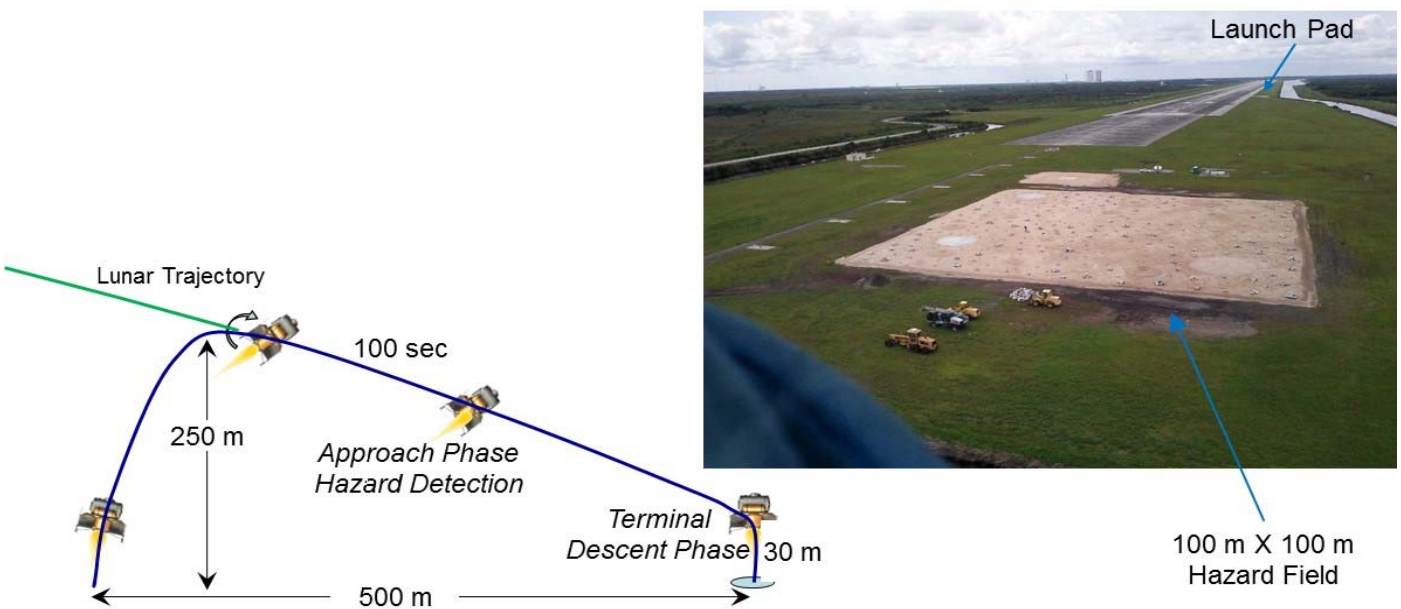

Figure 6. Flight profile for demonstrating autonomous safe landing at a hazard field (simulated lunar terrain) constructed at north end of Shuttle Landing Facility.

As shown in Fig. 5, the flash lidar head is mounted to a 2-axis gimbal to point the lidar at the hazard field (targeted landing area) and to execute a raster scan pattern that allows mapping the whole field. The lidar receiver field-Of-View (FOV) was chosen to be $1.0 \mathrm{deg}$. to allow for $10 \mathrm{~cm}$ Ground Sample Distance (GSD) from a $750 \mathrm{~m}$ slant range to the target site. Prior analysis indicated that a maximum GSD of $10 \mathrm{~cm}$ is required for reliable detection of hazards of $40 \mathrm{~cm}$ in dimensions given a lidar range precision of $8 \mathrm{~cm}^{4}$. The gimbal, controlled by the HDS using the vehicle position and attitude, ensured a series of overlapping lidar image frames that can be stitched together to create a $100 \mathrm{~m}$ x $100 \mathrm{~m}$ Digital Elevation Map (DEM). About one hundred lidar flashes is required to produce a $100 \mathrm{~m}$ x $100 \mathrm{~m}$ DEM with the current 128x128 pixels lidar. All the processing of the flash lidar range images were performed, close to real-time, onboard Morpheus by the HDS to generate a DEM, identify the safe landing locations, select the most suitable site, and provide its coordinate to the navigation system. Fig. 7 shows two examples of the flash lidar data from the Morpheus flights. One is a range image from a slant path angle of $30^{\circ}\left(60^{\circ}\right.$ angle of incidence for the lidar) showing some rock piles and the ground slope (color gradient from bottom to top) as apparent from the lidar view angle. Second is a derived elevation image showing rocks as small as $30 \mathrm{~cm}^{19}$.
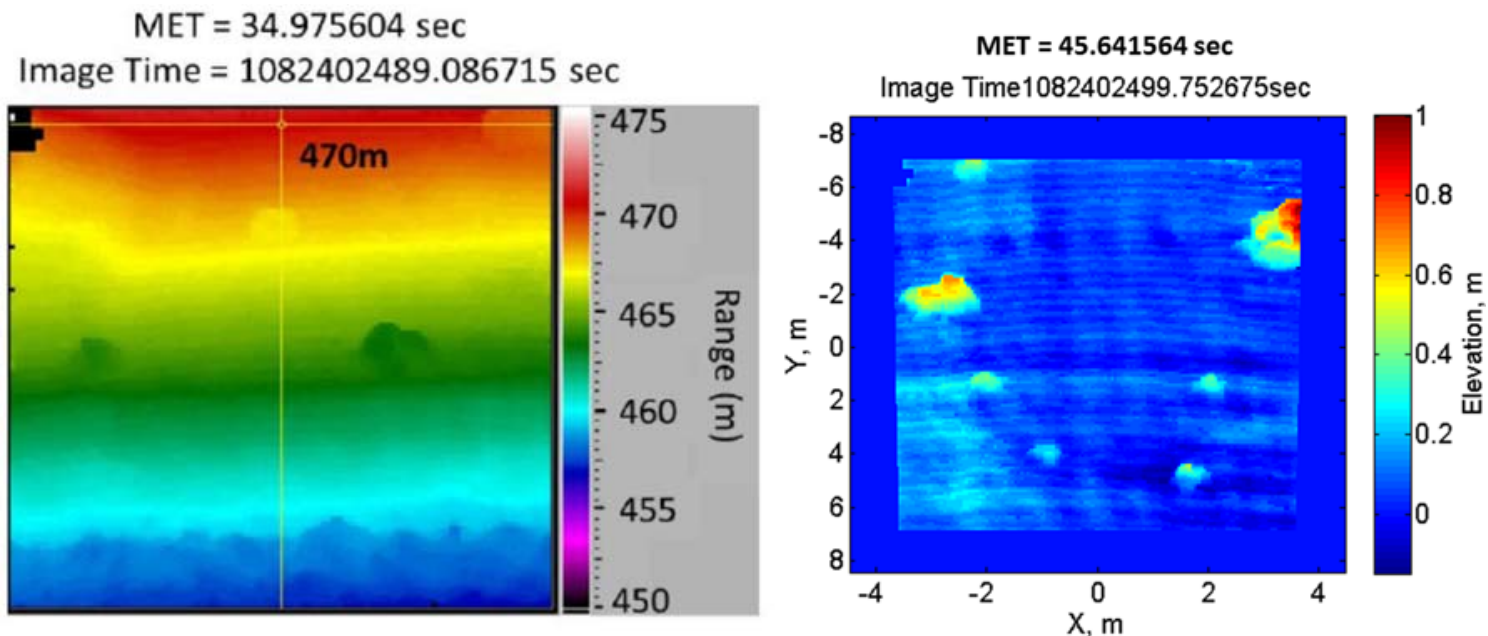

Figure 7. Examples of flash lidar data from Morpheus flight test. Range image on the left shows some rock piles and the ground slope (color gradient) as seen by the lidar, and the image on the right is a projected elevation image showing rocks as small as $30 \mathrm{~cm}$. 


\section{FLASH LIDAR TECHNOLOGY ADVANCEMENTS}

Thus far, our effort on flash lidar technology has been focused on assessing its capabilities in meeting NASA's needs and building a fully-autonomous and robust experimental unit that can operate reliably for field test campaigns and closedloop demonstration flight onboard Morpheus vehicle. After successful closed-loop flight tests, our focus is now shifting towards enhancing the flash lidar performance, optimizing its design, and addressing space qualification requirements. Table 2 lists the current ALHAT flash lidar specifications and the desired parameters that can satisfy the needs of landing missions to the solar system bodies (Moon, Mars, Saturn moons, etc.), asteroid missions (sample return and redirect), and AR\&D of space vehicles with other space vehicles, satellites, and international space station. A major driver in establishing the flash lidar advancement path is the need to map a $100 \mathrm{~m}$ x $100 \mathrm{~m}$ area with $10 \mathrm{~cm}$ resolution (GSD) that is a $1 \mathrm{M}$ pixels DEM. But, a flash lidar with a $1 \mathrm{M}$ pixels detector array may be impractical for foreseeable future. Such sensor will require significant advancements in Avalanche Photodetector (APD) arrays and associated Readout Integrated Circuit (ROIC) chip leading to a much smaller pitch size and considerably lower-noise and higher gain than the current state of technology in order to allow for a reasonable laser pulse energy and receiver aperture size. A near-term compromise could be a 100kclass detector array and use of multiple image frames to construct a 1M pixels DEM. There are two possible approaches for generating a DEM larger than the individual flash lidar images. First approach is scanning the lidar FOV over the target area to generate a mosaic of image frames that can be stitched together to produce a $1 \mathrm{M}$ pixels DEM. This approach was successfully implemented and demonstrated by ALHAT in Morpheus flight test using the current 16K pixels lidar ${ }^{20}$. The second approach is Super-Resolution (SR) technique for which the lidar FOV is enlarged to cover the whole area and then blend a sequence of image frames of the same scene to achieve the desired resolution. Compared with mosaicking, this technique, as described in next section, has the advantage of smaller gimbal (only used for pointing), improved DEM quality (lower noise and eliminated bad pixels), and reduced acquisition time (fewer image frames). In addition, the SR algorithm provides independent relative position and orientation information that can help with precision navigation during final approach phase of landing and AR\&D maneuvers. A description of SR technique and its capabilities are provided in next section.

Table 2. Flash lidar specifications satisfying both landing and A\&RD applications compared with current instrument.

\begin{tabular}{|l|c|c|}
\hline Parameter & Current Flash Lidar & Goal \\
\hline Detector Arrav Size & $16 \mathrm{~K}$ & $100 \mathrm{k}$ \\
\hline Range Precision within a frame $(1-\sigma)$ & $7 \mathrm{~cm}$ & $3 \mathrm{~cm}$ \\
\hline Frame Rate & $20 \mathrm{~Hz}$ & $20 \mathrm{~Hz}$ \\
\hline Operational Wavelength & 1.06 micron & $\begin{array}{l}1.06 \text { micron, eye-safe } 1.57 \\
\text { micron for human missions }\end{array}$ \\
\hline $\begin{array}{l}\text { Max Operational Range (for diffuse } \\
\text { target with 30\% reflectivity at } \\
\text { normal look angle) }\end{array}$ & $1800 \mathrm{~m}$ & $3000 \mathrm{~m}$ \\
\hline
\end{tabular}

\section{SUPER RESOLUTION ALGORITHM}

The SR technique takes advantage of sub-pixel shifts between multiple, low-resolution images of the same scene to construct a higher resolution image. SR is a well-established technique for enhancing two-dimensional (2-D) images and over the years, a large number of algorithms have been developed for processing intensity images produced by different types of imaging systems ${ }^{21,22}$. With emergence of flash lidar technology, the application of SR technique was proposed by a few workers. However, these earlier efforts could only achieve 3-D image enhancements when the camera was subjected to a controlled motion within a tightly constrained envelope, with or without image registration ${ }^{23-26}$. The requirement to tightly constrain camera motion in these techniques precluded their implementation in 3-D cameras installed on many surface, airborne, and space-based platforms since those platforms typically undergo significant excursions in position and orientation. An additional limitation of the cited works is that they require external sensors to provide the camera's position and pointing angle. We have developed and demonstrated a 3-D SR algorithm that creates a DEM with a digital 
magnification factor of four to eight in real-time while providing all six components of the lidar's position and orientation vector $^{27}$.

The SR algorithm, as illustrated in Fig. 8, is based on three major blocks: 3-D modification of the back-projection method ${ }^{25}$, a 6-D version of the Lucas-Kanade registration algorithm ${ }^{28}$, and the modified inverse filtering algorithm ${ }^{29}$. A backprojection method has recently been applied to solve the super-resolution problem in the 2-D environment ${ }^{25}$. We developed a 3-D implementation of the of the back-projection algorithm that leads to reconstruction of a 3-D surface for an arbitrary look-angle using an iteration process that allows for a real time code. The 6-D registration algorithm computes the sixdegree-of-freedom relative state vector (lidar instrument position coordinates and three components of pointing angle) using consecutive image frames. Determination of the instrument state vector is critical for registration of individual frames prior to combining them to generate a SR image using the inverse filter algorithm. The state vector data provided by this algorithm can also be used by the instrument platform to accurately navigate to the intended destination.

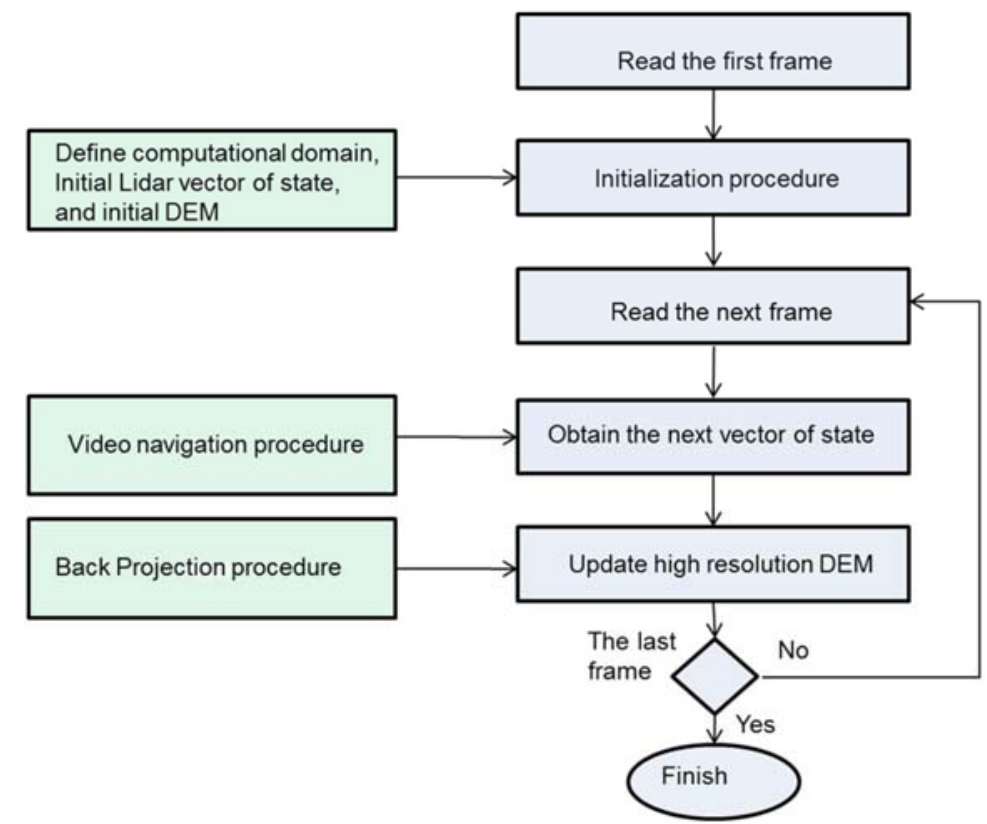

Figure 8. Super-Resolution Algorithm providing high resolution DEM and instrument's 6-DOF relative state vector.

Performance of the SR was analyzed using a high fidelity Mathlab model showing that a digital magnification factor of four to eight (16X to $64 \mathrm{X}$ number of original image pixels) can be achieved by processing 20 consecutive flash lidar frames. The simulations were based on current ALHAT flash lidar characteristics and realistic platform motions. It was also shown that a modest improvement in the flash lidar range noise within the frame and shot-to-shot range precision can provide a digital magnification of eight on consistent basis using less than 20 frames. The accuracy of the Mathlab model was later verified by actual flash lidar data from a helicopter flight test in a real-time SR processor.

The SR algorithm was implemented on two different processing platform: a high-speed Vertex 5 FPGA (FieldProgrammable Gate Array) and a graphics processing unit (GPU). Neither of these SR processor has been integrated into the ALHAT flash lidar yet. But, their operation has been demonstrated by inputting the recoded data from one of the helicopter flights during Field Test 5 campaign at NASA Kennedy Space Center (see Fig. 4). The flight was conducted over the well-surveyed hazard field (Fig. 6) with $10 \mathrm{~cm}$ spatial and range resolution. Fig. 9 shows the truth DEM of the hazard field showing its surface contour, rock piles, and craters. The flight followed a trajectory from $1 \mathrm{~km}$ slant range to $50 \mathrm{~m}$ from the field at a look angle close to 30 degrees. A 5 degree FOV lens was used for this flight to cover a relatively large area of the hazard field.

Fig. 10 provides an example of the helicopter flight results obtained from $400 \mathrm{~m}$ slant range. In addition to noise suppression and improving spatial resolution, this example demonstrates another super-resolution attribute, namely the ability to fill in missing data by combining information from several frames. The DEM obtained from a single frame (Fig.10 a) contains many blank spots. These "bad pixels" are basically non-responding detectors. Most of these spots are 
compensated by data recovered from other frames as the bad pixels move spatially to adjacent portions of the target, thus exposing the area that they previously blocked. Fig. 11 demonstrates how small scale-structures become identifiable and the image noise is reduced. The extended area 2 as shown in Fig. 12 demonstrates that the hazards (rocks) with a diameter $0.4 \mathrm{~m}$ and height $0.2 \mathrm{~m}$ can be identified.

Comparing the true DEM with the restored SR DEM, as illustrated in Fig. 13, reveals the merits of the SR image enhancement technique. The black rectangular on the true DEM shows position and orientation of the restored DEM. All large scale features like big craters and rocks are very visible and located in the same positions as they are located in the true DEM. Six small features (rocks) are compared. The arrows in Fig. 13 point to pairs of rocks on both DEM's. Table 3 lists the measured diameters and heights of the six rocks from the truth map and compares them with the measurements obtained from the SR processed flash lidar data. The diameter is defined as the average size in two horizontal orthogonal directions close to a rock base and height is defined as the highest point. Five of the six rocks can be identified with high certainty. One which cannot be identified is smaller than the $30 \mathrm{~cm}$ threshold goal.

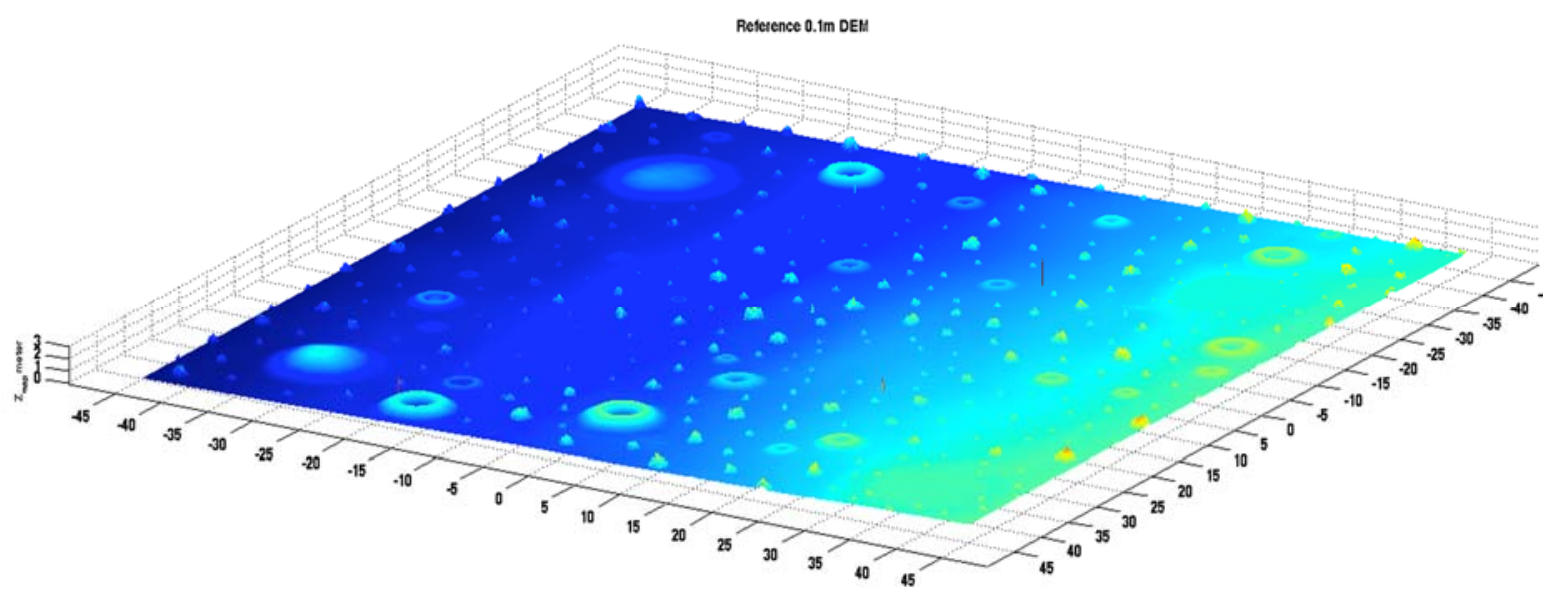

Figure 9. Truth DEM of hazard field with $10 \mathrm{~cm}$ resolution.
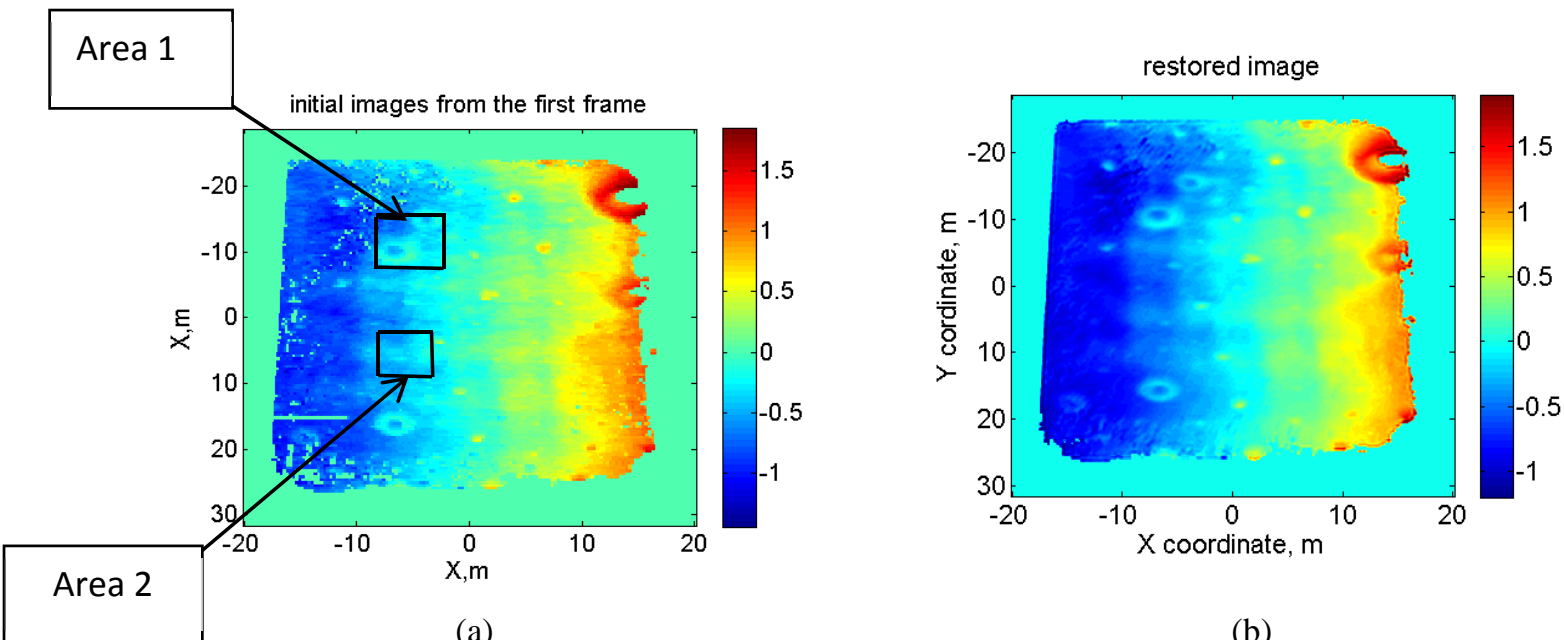

(a)

(b)

Figure 10. Results of DEM restoration: a) DEM obtained using 1 frame; b) SR DEM obtained from 20 frames. 


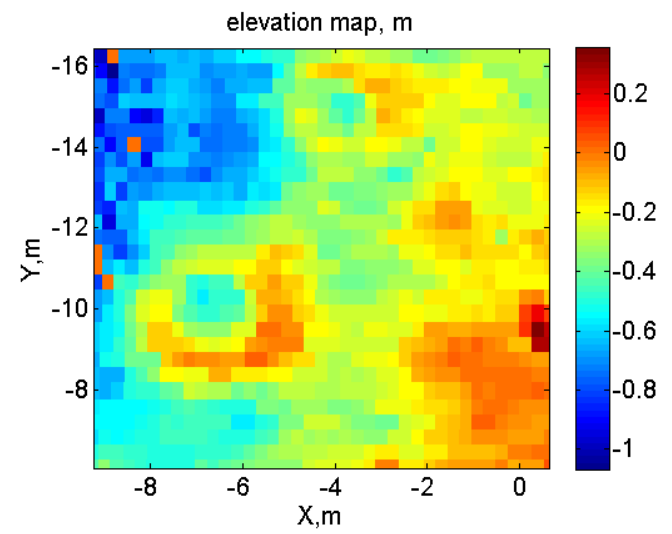

(a)

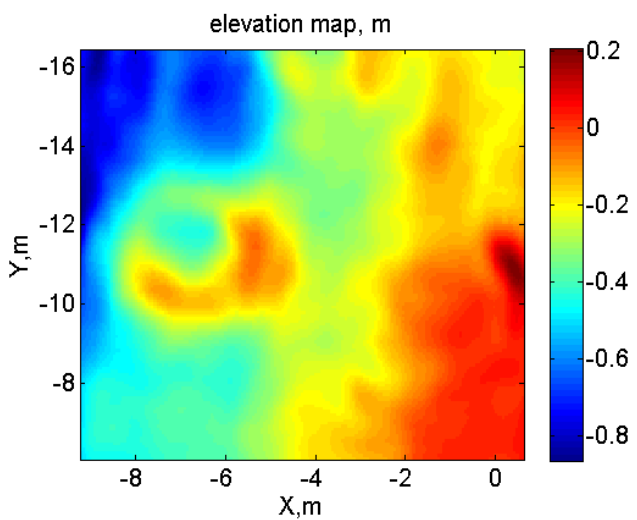

(b)

Figure 11. Zoomed Area 1 (from Figure 17): a) DEM obtained from 1 frame; b) SR DEM obtained from 20 frames.

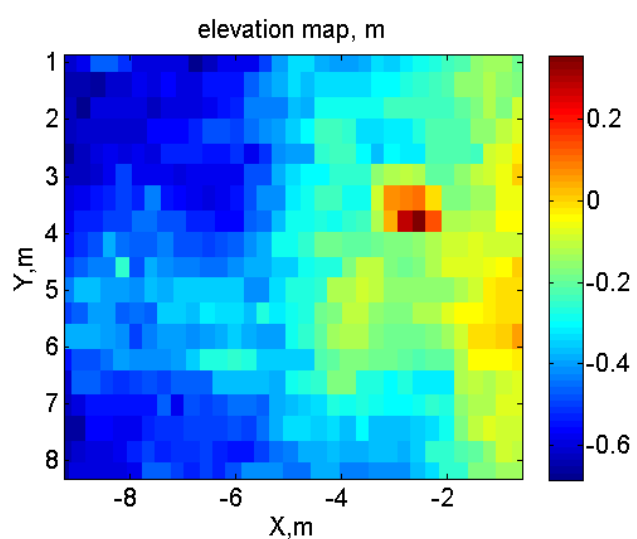

(a)

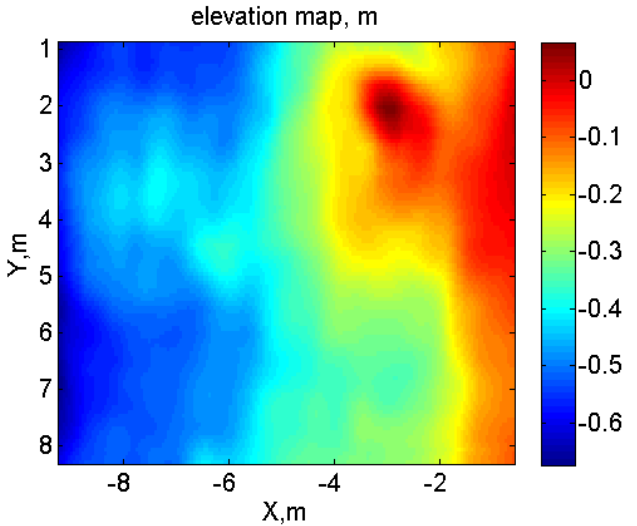

(b)

Figure 12. Zoomed Area 2 (from Figure 17): a) DEM obtained from 1 frame; b) SR DEM obtained from 20 frames.

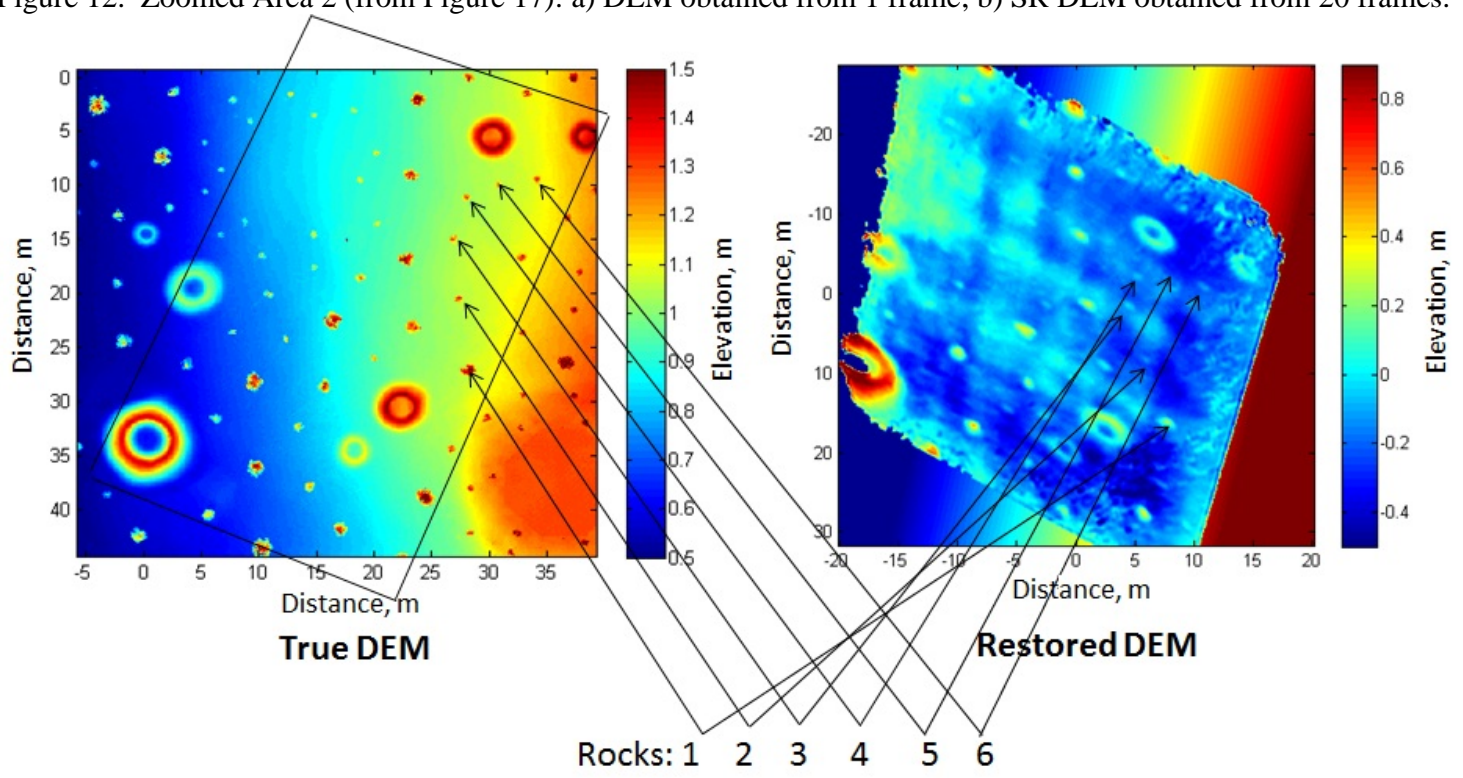

Figure 13. Comparison of the truth DEM and SR restored DEM. 
Table 3. Results of helicopter flight test.

\begin{tabular}{|c|c|c|c|c|}
\hline \multirow{2}{*}{$\begin{array}{c}\text { Rock } \\
\text { Number }\end{array}$} & \multicolumn{2}{|c|}{ Diameter (m) } & \multicolumn{2}{c|}{ Height (m) } \\
\cline { 2 - 5 } & True & Restored & True & Restored \\
\hline 1 & 1.3 & 1.3 & 0.6 & 0.5 \\
\hline 2 & 0.8 & 1.2 & 0.3 & 0.12 \\
\hline 3 & 0.7 & 1.0 & 0.4 & 0.21 \\
\hline 4 & 0.6 & 1.0 & 0.2 & 0.3 \\
\hline 5 & 0.6 & - & 0.25 & - \\
\hline 6 & 0.7 & 0.6 & 0.27 & 0.2 \\
\hline
\end{tabular}

The ability of the SR algorithm in determining the vehicle 6-DOF state vector is demonstrated in Fig.14 showing the vehicle altitude obtained from SR algorithm without input from other sensors. Plot on the left compares the flash lidar SR altitude with that of the ALHAT navigation system. The difference is mostly due to different definition of altitudes and the slope of terrain. The plot on the right compares the SR altitude with the Doppler lidar data ${ }^{15}$. The mean and standard deviation of the difference between two measurements is only about $0.5 \mathrm{~m}$ and $1.0 \mathrm{~m}$ respectively. These values are well within the Doppler lidar error and the terrain slope and roughness.

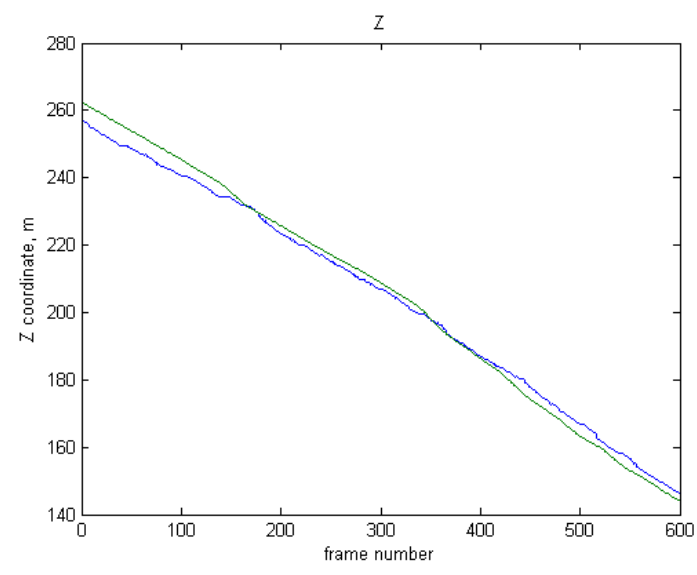

(a)

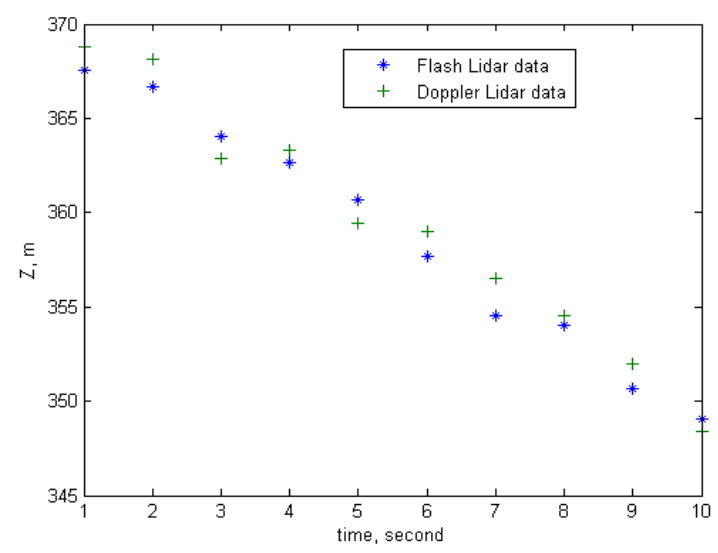

(b)

Figure 14. Altitude data obtained by applying SR algorithm to flash lidar data from the helicopter flight test: a) Compared with ALHAT navigation system data; b) Compared with Doppler lidar data.

\section{CONCLUSION}

The flash lidar is recognized by NASA as a key enabling technology for autonomous safe precision landing on solar system bodies (Moon, Mars, Jupiter and Saturn moons, etc.), and autonomous rendzevous and docking necessary for asteroid sample return and redirct missions, spacecraft docking, satellite servicing, and space debris removal. The ALHAT project developed and demonstrated a flash lidar sensor system for landing application through closed-loop Morpheus flight test campaign. The autonomous rendzevous and docking application of flash lidar was previously assessed by three Space Shuttle missions to International Space Station. These programs revealed the impact of flash lidar technology on NASA's operation in earth orbit and exploration missions beyond the earth orbit. However, the flash lidar in its current state does not fully meet the desired performance specifications for neither landing nor rendzevous and docking applications. A set of common specifications has been defined to direct the flash lidar technology advancement toward a single sensor that 
satisfies a wide spectrum of landing missions and missions requiring autonomous rendzevous and docking capabilities. Such a flash lidar sensor can be developed in near-term by a combination of an incrementaly larger focal plabe array with an order of $100 \mathrm{k}$ pixels and super-resolution algorithm to produce sufficiently large digital elevation maps with $1 \mathrm{M}$ to $6 \mathrm{M}$ pixels from a range of the order of $3 \mathrm{~km}$.

\section{ACKNOWLEDGMENTS}

The authors are grateful Chirold Epp, NASA Johnson Space Center, for his guidance and support for almost 10 years as the ALHAT project manager. We also would like to thank Edward Robertson, current ALHAT project manager and NASA's Advanced Exploration Systems (AES) program office for their continued support. The authors also acknowledge the ALHAT and Morpheus team members from the NASA Johnson Space Center and the NASA Jet Propulsion Laboratory for their collaboration and the NASA Kennedy Space Center for facilitating the field tests.

\section{REFERENCES}

1. Epp, C. D., Robinson, E. A., and Brady, T., “Autonomous Landing and Hazard Avoidance Technology (ALHAT)”, Proc. of IEEE Aerospace Conference, paper no. 1644, 2008.

2. Bo J. Naasz and Michael C. Moreau, “Autonomous RPOD Challenges for the Coming Decade,” Proc. of 35th Annual American Astronautical Society Guidance and Control Conference, 2012.

3. Johnson, A.E., and Montgomery, J., “An Overview of Terrain Relative Navigation for Precise Lunar Landing,” IEEE Aerospace Conference, 2008.

4. Huertas, A., Johnson, A. E, Werner, R. A., Maddock, R A., "Performance Evaluation of Hazard Detection and Avoidance Algorithms for Safe Lunar Landings,” Proc. IEEE Aerospace Conference, PP 1-20, 2010.

5. Amzajerdian, F., Vanek, M. D., Petway, L. B., Pierrottet, D. F., Busch,G. E., Bulyshev, A., "Utilization of 3-D Imaging Flash Lidar Technology for Autonomous Safe Landing on Planetary Bodies,“ SPIE Proceeding Vol. 7608, paper no 80, 2010.

6. B. Barbee, J. Carpenter, S. Heatwole, F. Markley, M. Moreau, B. Naasz, and J. V. Eepoel, “Guidance and Navigation for Rendezvous and Proximity Operations with a Non-Cooperative Spacecraft at Geosynchronous Orbit,” Proc. of the AAS George H. Born Symposium, 2010.

7. J. A. Christian, H. Hinkel, C. N. D’Souza, S. Maguire, and M. Patangan, "The Sensor Test for Orion RelNav Risk Mitigation (STORRM) Development Test Objective,” Proc. of AIAA Guidance, Navigation, and Control Conference, No. AAS 11-6260, 2011.

8. Stettner, R., Bailey, H., and Silverman, S., "Three Dimensional Flash Ladar Focal Planes and Time Dependent Imaging,” International Symposium on Spectral Sensing Research, Bar Harbor, Maine, 2006.

9. Stettner, R., “Compact 3D Flash LIDAR video cameras and applications,” Proc. of SPIE Vol. 7684, 768405, 2010.

10. Farzin Amzajerdian, Diego Pierrottet, Larry Petway, Glenn Hines, Vincent Roback and Robert Reisse, "Lidar Sensors for Autonomous Landing and Hazard Avoidance," Proc. of AIAA Space and Astronautics Forum and Exposition, 2013.

11. Brady, T., Schwartz, J., and Tillier, C., "System Architecture and Operational Concept for an Autonomous Precision Lunar Landing System," AAS 30th Rocky Mountain Guidance and Control Conference, 2007.

12. Trawny, N., Carson, J. M., Huertas, A., Luna, M. E., Roback, V. E., Johnson, A. E., Martin, K. E., and Villalpando, C. Y., "Helicopter Flight Testing of a Real-Time Hazard Detection System for Safe Lunar Landing," Proc. AIAA SPACE Conference \& Exposition, 2013.

13. Epp, C., Robertson, E., and Brady, T., "Autonomous Landing and Hazard Avoidance Technology (ALHAT)," Proc. IEEE Aerospace Conference, 2008.

14. Roback, V. E., Bulyshev, A., Amzajerdian, F., Brewster, P. F., Barnes, B. W., Kempton, K. S., and Reisse, R. E., "Helicopter Flight Test of 3-D Imaging Flash LIDAR Technology for Safe, Autonomous, and Precise Planetary Landing,” Proc. of SPIE ,Vol. 8731, SPIE Defense Security, and Sensing Conference, 2013. 
15. Pierrottet, D. F., Amzajerdian, F., Petway, L. B., Barnes, B. W., Lockard, G., and Hines, G. D., "Navigation Doppler Lidar Sensor for Precision Altitude and Vector Velocity Measurements Flight Test Results,” Proceeding SPIE Vol. 8044, 2011.

16. Project Morpheus Website http://morpheuslander.jsc.nasa.gov

17. John M.Carson, Edward A. Robertson, Diego F. Pierrottet, Vincent E. Roback, Nikolas Trawny, Jennifer L. Devolites, Jeremy J. Hart, Jay N. Estes, Gregory S. Gaddis, "Preparation and Integration of ALHAT Precision Landing Technology for Morpheus Flight Testing,” Proc. AIAA SPACE Conference and Exposition, 2014.

18. Rutishauser, D., Epp, C. D., and Robertson, E. A., "Free-Flight Terrestrial Rocket Lander Demonstration for NASA's Autonomous Landing and Hazard Avoidance Technology (ALHAT) System,” Proc. of AIAA SPACE, 2012.

19. Vincent E. Roback, Diego F. Pierrottet, Farzin Amzajerdian, Bruce W. Barnes, Glenn D. Hines, Larry B. Petway, Paul F. Brewster, Kevin S. Kempton, and Alexander E. Bulyshev, "Lidar sensor performance in closed-loop flight testing of the Morpheus rocket-propelled lander to a lunar-like hazard field,” Proc. of AIAA Science and Technology Forum and Exposition, 2015.

20. Nikolas Trawny, Andres Huertas, Michael Luna, Carlos Y. Villalpando, Keith E. Martin, John M. Carson III, Andrew E. Johnson, Carolina Restrepo, Vincent E. Roback, "Flight testing a Real-Time Hazard Detection System for Safe Lunar Landing on the Rocket-powered Morpheus Vehicle,” Proc. of AIAA Science and Technology Forum and Exposition, 2015.

21. S. Park, M. K. Park, and M. Kang, "Superresolution image reconstruction: A technical review,” IEEE Signal Processing Magazine 20(3), 21-36, 2003.

22. S. Young and R. Driggers, "Superresolution image reconstruction from a sequence of aliased imagery," Applied Optics 45(21), 5073-5085, 2006.

23. G. Rosenbush, T. Hong, and R. Eastman, "Super-resolution enhancement of flash LADAR range data," in Proceedings of SPIE, Unmanned/Unattended Sensors and Sensors Networks IV, 6736, 673614-1 - 673614-10, (2007)

24. S. Hu, S. S. Young, T. Hong, J. Reynolds, K. Krapels, B. Miller, J. Thomas, and O. Nguyen, "Super resolution for flash LADAR imagery,” Applied Optics 49(5),772-780, 2010.

25. S. Hu, S. Young, T. Hong, J. Reynolds, K. Krapels, B. Miller, J. Thomas, and O. Nguyen, "Super-Resolution for flash LADAR data,” Proceedings of SPIE vol. 7300, 2009.

26. J. Woods, E. Armstrong, W. Armbruster, and R. Richmond, "The application of iterative closest point (ICP) registration to improve 3D terrain mapping estimates using the flash 3D ladar system,” Proceedings of SPIE vol. 7684, 2010.

27. Bulyshev, Alexander; Amzajerdian, Farzin; Roback, Vincent E; Hines, Glenn; Pierrottet, Diego; Reisse, Robert, "Three-dimensional super-resolution: theory, modeling, and field test results," Applied Optics, Vol. 53 Issue 12, pp.2583-2594, 2014.

28. B. Lucas, T. Kanade, “An Iterative Image Registration Technique with Application to Stereo Vision,” Proceedings of Imaging Understanding Workshop, 121-130, 1981.

29. E. Armstrong, R. Richmond, “The application inverse filter to 3D Microscanning of LADAR Imagery,” Proceedings of IEEE Aerospace Conference, 2006. 